\title{
A global time integration approach for realistic unsteady flow computations
}

\author{
Peter Eliasson \\ FOI, Swedish Defence Research Agency, SE-16490 Stockholm, Sweden \\ Tomas Lundquist, Jan Nordström \\ Linköping University, SE-581 83, Linköping, Sweden
}

\begin{abstract}
A novel time integration approach is explored for unsteady flow computations. It is a multi-block formulation in time where one solves for all time levels within a block simultaneously. The time discretization within a block is based on the summation-by-parts (SBP) technique in time combined with the simultaneous-approximation-term (SAT) technique for imposing the initial condition. The approach is implicit, unconditionally stable and can be made high order accurate in time. The implicit system is solved by a dual time stepping technique. The technique has been implemented in a flow solver for unstructured grids and applied to an unsteady flow problem with vortex shedding over a cylinder. Four time integration approaches being $2^{\text {nd }}$ to $5^{\text {th }}$ order accurate in time are evaluated and compared to the conventional $2^{\text {nd }}$ order backward difference (BDF2) method and a $4^{\text {th }}$ order diagonally implicit Runge-Kutta scheme (ESDIRK64). The obtained orders of accuracy are higher than expected and correspond to the accuracy in the interior of the blocks, up to $8^{\text {th }}$ order accuracy is obtained. The influence on the accuracy from the size of the time blocks is small. Smaller blocks are computationally more efficient though, and the efficiency increases with increased accuracy of the SBP operator and reduced size of time steps. The most accurate scheme, with a small time step and block size, is approximately as efficient as the ESDIRK64 scheme. There is a significant potential for improvements ranging from convergence acceleration techniques in dual time, alternative initialization of time blocks, and by introducing smaller time blocks based on alternative SBP operators.
\end{abstract}

\section{Introduction}

A majority of fluid dynamics problems in engineering applications are inherently unsteady. These flow phenomena include e.g. transition, turbulent unsteady flow separation, unsteady boundary layers and shear-flows. To accurately predict these phenomena, accurate and efficient time integration is required. For realistic applications the time integrator has to be implicit since the restriction on the time step from an explicit approach on highly stretched grids is typically several orders of magnitude smaller than the temporal resolution required to resolve the time dependent flow physics.

The development of efficient time integration strategies for unsteady flows have so far mostly been restricted to one-step schemes, where an equation system must be solved at each time level. Such schemes include linear multistep methods as well as diagonally implicit Runge-Kutta methods, and are straightforward to implement efficiently using a standard multigrid or Newton iteration scheme. Fully global implicit methods, on the other hand, have generally not been considered, despite their excellent accuracy and stability properties.

In this work we explore an essentially global time integration approach, based on summation-by-parts (SBP) operators together with weak simultaneous-approximation-term (SAT) initial conditions. This method was developed in ${ }^{1,2}$ and forms a natural extension to the SBP-SAT technique in space ${ }^{3-7}$, known for its optimal discrete energy properties. An initial efficiency study of SBP-SAT schemes in time was done in ${ }^{8}$ for a simple boundary layer model problem, employing a standard multigrid iteration technique with dual time stepping to solve the implicit equation systems.

The semi-global approach taken here, however, means that we solve the system for a large number of time levels simultaneously. To ensure fast convergence in this setting, we develop an extension of the dual time multigrid iteration technique. We implement this new method in a flow solver for unstructured grids. The efficiency of the 
semi-global schemes are investigated on an inherently unsteady flow case and the results are compared to the efficiency of other more conventional time integrators.

In the following sections the summation by parts technique in time is introduced and applied to the NavierStokes equations. Then follows a description of the steady state problem in dual time, the flow solver Edge for unstructured grids and a short description of the implementation of the approach. Next, numerical results are presented for a model problem and the flow over a cylinder followed by a discussion of the results.

\section{Integration by summation-by-parts}

The time dependent equations considered are the semi-discretized Navier-Stokes equations where a finitevolume discretization is applied to the space derivatives. This results in a massive set of ordinary differential equations (ODE's),

$$
\frac{\partial q}{\partial t}+R(q)=0 ; \quad q(t=0)=f
$$

which will be integrated in time. In Eq. (1) $q$ is the unknown state vector, consisting of conservative discrete flow variables, the vector $R(q)$ results from the semi-discretization of the Navier-Stokes equations involving convection, diffusion and source terms for the turbulence equations.

Let $t=(0, \Delta t, 2 \Delta t, \cdots,(N-1) \Delta t=T)$ be a uniform vector in time with $N$ discrete time levels. An SBP-SAT approximation to Eq. (1) can then be written as

$$
\left(D \otimes I_{R}\right) \vec{q}+R(\vec{q})=P^{-1} \sigma \vec{e}_{0} \otimes\left(q_{0}-f\right)
$$

where $\vec{q}=\left(q_{0}, q_{1}, q_{2}, \cdots, q_{N-1}\right) \quad$ contains the solution vectors at the different time levels, $R(\vec{q})=\left(R\left(q_{0}\right), R\left(q_{1}\right), \cdots, R\left(q_{N-1}\right)\right)$ contains the residuals of the solution vectors at the same time levels. Note that $q_{i}$ is a vector with all variables in space with $N_{E q} N_{S}$ unknowns at time level $i \Delta t, R\left(q_{i}\right)$ is its residual vector, $N_{E q}$ is the number of governing equations and $N_{S}$ is the total number of nodes in the computational domain. The matrix $I_{R}$ is the identity matrix of size $N_{E q} N_{S} \times N_{E q} N_{S}$, and $\vec{e}_{0}=(1,0, \cdots, 0)$ is the first unit vector in $\mathfrak{R}_{N+1}$. The Kronecker product ${ }^{5} \otimes$ is a special type of matrix multiplication. The SAT penalty treatment on the right hand side of Eq. (2) forces the initial solution $q_{0}$ towards initial data $f$. The penalty coefficient $\sigma$ is set to $\sigma=-1$ which is the optimal choice in terms of accuracy, and additionally leads to sharp fully discrete energy estimates. ${ }^{1,2,8}$

As the first derivative $D$ in Eq. (2) we use diagonal norm finite difference operators on summation-by-parts form. This form is characterized by the factorization $D=P^{-1} Q$, where $P$ is a diagonal, positive definite quadrature matrix, and $Q$ is an almost skew-symmetric matrix defined by $Q+Q^{T}=\vec{e}_{N-1} \vec{e}_{N-1}^{T}-\vec{e}_{0} \vec{e}_{0}^{T}=E_{N-1}-E_{0}$, that is based on a repeated central difference stencil in the interior. One example is the classical second order central difference approximation,

$$
P=\Delta t\left[\begin{array}{ccccc}
1 / 2 & & & & \\
& 1 & & & \\
& & \ddots & & \\
& & & 1 & \\
& & & & 1 / 2
\end{array}\right], \quad Q=\left[\begin{array}{ccccc}
-1 / 2 & 1 / 2 & & & \\
-1 / 2 & 0 & 1 / 2 & & \\
& & \ddots & & \\
& & -1 / 2 & 0 & 1 / 2 \\
& & & -1 / 2 & 1 / 2
\end{array}\right],
$$

with first order one-sided differences at the boundaries of the operator (first and last time levels), and where $P$ represents the second order trapezoidal rule. Higher order operators of the same type can be constructed, with order $2 \mathrm{~s}$ in the interior, order $\mathrm{s}$ at the boundaries, and order $2 \mathrm{~s}$ for the diagonal quadrature norms, for $\mathrm{s}=1,2, \ldots, 10$. When used in an SBP-SAT approximation of initial value problems, these operators lead to time 
integration schemes of high order (2s), moderate stage order (s), and optimal stability properties also for non-linear problems (A-, L- and B-stability, with sharp fully discrete energy estimates). ${ }^{7}$ We denote these schemes $\operatorname{SBP}(2 \mathrm{~s}, \mathrm{~s})$. Here we explore four SBP schemes, $s=1-4$, and denote the schemes $\operatorname{SBP}(2,1), \operatorname{SBP}(4,2), \operatorname{SBP}(6,3), \operatorname{SBP}(8,4)$, being globally $2^{\text {nd }}, 3^{\text {rd }}, 4^{\text {th }}$ and $5^{\text {th }}$ order accurate in time, respectively. Some of the corresponding matrices $P, Q$ are given in appendix. We require time blocks with at least one interior node with a central difference stencil which restricts the minimum size $N$ of the matrices to $N \geq 3, N \geq 9, N \geq 13, N \geq 17$ for the four SBP schemes, respectively. Furthermore, given the optimal value of the penalty coefficient $\sigma=-1$ used, the order of accuracy in the last time level in each block will be 2 s, i.e. the same order as for the schemes in the interior, and hence higher than at other time levels in the boundaries of the blocks being of order $\mathrm{s}+1 .{ }^{2,8,13}$

It should also be noted that many other types of SBP operators exist, see ${ }^{9}$ for a comprehensive review which also includes a generalized approach. It has also been shown that the resulting time integration schemes form a subset of implicit Runge-Kutta schemes. ${ }^{10-12}$ In this work we focus on the large classical finite difference based operators described above. The total size of the fully discrete system becomes $(N+1) \times N_{E q} N_{S} \times N_{E q} N_{S}$. For long calculations in time, it may still be necessary to divide the time interval into a smaller number of subintervals, or time blocks, in order to reduce $N$ and thus the size of the implicit equation system. This poses no problem, since all the relevant properties of the SBP-SAT technique are also valid in a multi-stage approach. ${ }^{8}$ In that case we have the sequence of problems

$$
\left(D \otimes I_{R}\right) \vec{q}^{l}+R\left(\vec{q}^{l}\right)=P^{-1} \sigma \vec{e}_{0} \otimes\left(q_{0}^{l}-q_{N-1}^{l-1}\right)
$$

where the superscript $l$ indicates the solution on time interval number $l$ given by $t \in\left(T_{l-1}, T_{l}\right)$, where each interval denotes a time block, $T_{i}=i \Delta t N, i=[0,1, \cdots, N-1]$. Note that the solution $q_{N-1}^{l-1}$ at the last time level of the previous interval $t \in\left(T_{l-2}, T_{l-1}\right)$ is used as initial data for the problem on $\left(T_{l-1}, T_{l}\right)$. For the first interval, we use the definition $q_{N-1}^{0}=f$ to impose the physical initial condition. In this way, the size of the implicit systems to solve Eq. (4) remains constant (but still potentially large) for arbitrary simulation times.

\section{The steady-state problem}

\section{A. Dual time stepping}

The SBP-SAT implicit scheme in Eq. (2) can, in general, not be solved directly since $R$ is a non-linear function of the solution variables $\vec{q}$ in space and time. Hence an iterative procedure is required. We introduce an artificial time derivative in dual time $\tau$ and rewrite Eq. (2) as

$$
\frac{d \vec{q}}{d \tau}+\tilde{R}(\vec{q})=0 ; \quad \tilde{R}(\vec{q})=\left(P^{-1} \tilde{Q} \otimes I_{R}\right) \vec{q}+R(\vec{q})-\vec{F}
$$

where $\tilde{Q}=Q-\sigma E_{0}$ and $\vec{F}=P^{-1} \sigma \vec{e}_{0} \otimes f$ is a constant independent of $\vec{q}$. Provided that $d \vec{q} / d \tau \rightarrow 0$, the original equation Eq. (2) is retained. Note that the solution vector $\vec{q}$ contains the flow solutions for all $N$ time levels, i.e. it contains the entire solution vector in both space and time. Note also that the first term in $\tilde{R}(\vec{q})$ introduces the coupling between the $N$ solutions in time.

Explicit time marching in dual time will be used to march Eq. (5) towards steady state. The implementation is done in a flow solver that, originally, solves for the steady state of $R(\vec{q})$ using local time stepping. To ensure stability, the time steps are determined from estimations of the convective and diffusive spectral radius of $R(\vec{q})$, where the resulting time step is based on the maximum spectral radius, i.e.

$$
\Delta \tau=\min \left(\frac{C F L_{I}}{\rho_{I}(R)}, \frac{C F L_{V}}{\rho_{V}(R)}\right)
$$


where $\rho_{I}(R), \rho_{V}(R)$ denote the maximum eigenvalues of $R(q)$ along the imaginary and negative real axis, and $C F L_{I}, C F L_{V}$ the $C F L$ numbers along the same axis, respectively.

For the solution of Eq. (5), the additional term $\left(P^{-1} \tilde{Q} \otimes I_{R}\right) \vec{q}$ connecting the various time levels has to be accounted for to ensure stability in dual time. We do this by estimating the largest eigenvalues of $P^{-1} \tilde{Q}$ and add these to the spectral radius $\rho_{i}(R), \rho_{v}(R)$. Since the penalty coefficient is $\sigma=-1$ the eigenvalues have negative real parts ${ }^{2}$ and the maximum real and imaginary eigenvalues vary with the size $N$ of $P^{-1} \tilde{Q}$ as well with the specific operator. The largest eigenvalues are given in Table 1 for the three considered operators here given in appendix. The largest real eigenvalues are reduced as the size of $N$ increase whereas the largest imaginary eigenvalues increase with increasing size of $N$ and accuracy of $P^{-1} \tilde{Q}$.

Table 1. Largest eigenvalues along imaginary and negative real axis for various SBP operators. $N$ denotes the minimum size of the operator.

\begin{tabular}{lccc}
\hline Operator & $\mathbf{N}$ & $\Delta t \rho_{I}\left(P^{-1} \tilde{Q}\right)$ & $\Delta t \rho_{V}\left(P^{-1} \tilde{Q}\right)$ \\
\hline $\operatorname{SBP}(\mathbf{2 , 1})$ & $\geq 3$ & $\leq 1$ & $\leq 1$ \\
$\operatorname{SBP}(\mathbf{4 , 2})$ & $\geq 9$ & $<1.37$ & $<0.56$ \\
$\operatorname{SBP}(6,3)$ & $\geq 13$ & $<1.6$ & $<0.41$ \\
$\operatorname{SBP}(\mathbf{8 , 4 )}$ & $\geq 17$ & $<1.75$ & $<0.49$ \\
\hline \hline
\end{tabular}

\section{B. Artificial dissipation in time}

The SBP operators are of central differences with one sided differences at the boundaries. The choice of the penalty coefficient $\sigma=-1$ ensures that some numerical dissipation in time is introduced to the system. The steady state problem in Eq. (5) converges fast to a steady state for small block sizes. For larger blocks, however, we encounter convergence problems indicating that the amount of dissipation introduced by the initial condition is not sufficient. Our remedy is to introduce artificial dissipation in time, similar to what one typically does in space when central differences are used. To achieve this, we add an additional term to $\tilde{R}(\vec{q})$ in Eq. (5) with the artificial dissipation,

$$
\tilde{R}(\vec{q})=\left(P^{-1} \tilde{Q} \otimes I_{R}\right) \vec{q}+R(\vec{q})-\vec{F}+\varepsilon\left(P^{-1} \tilde{D}^{T} B \tilde{D} \otimes I_{R}\right) \vec{q}
$$

where $\tilde{D}$ is an undivided difference operator, $B$ a positive diagonal matrix and $\varepsilon$ a non-negative parameter that controls the amount of dissipation. This approach can be shown to be stable and the accuracy depends on the choice of the matrices $P, \widetilde{D}, B .{ }^{14}$ Note that $\widetilde{D}$ does not contain the time step $\Delta t$, it is included in $P$. We use matrices $\widetilde{D}$ based on $2^{\text {nd }}, 3^{\text {rd }}, 4^{\text {th }}, 5^{\text {th }}$ differences resulting in a dissipation with $4^{\text {th }}, 6^{\text {th }}, 8^{\text {th }}, 10^{\text {th }}$ difference stencils, respectively. These operators are $3^{\text {rd }}, 5^{\text {th }}, 7^{\text {th }}, 9^{\text {th }}$ order accurate, respectively. We employ a numerical dissipation operator that is higher than that of the SBP operator in the interior to avoid any influence from it on the overall accuracy. E.g., for the calculations with the $\operatorname{SBP}(6,3)$ scheme we employ a numerical dissipation based on $4^{\text {th }}$ difference operators $\widetilde{D}_{4}$ leading to a $7^{\text {th }}$ order accurate formulation and hence higher than that of the SBP scheme. Some of the employed operators and matrices are given in Appendix. All calculations use a moderate amount of dissipation, the amount is of the same size as the one typically employed for artificial dissipation in space.

\section{Implementation}

The CFD solver employed in the calculations is the Edge code, which is an edge- and node-based Navier-Stokes flow solver applicable for both structured and unstructured grids. ${ }^{15-17}$ Edge is based on a finite volume formulation where a median dual grid forms the control volumes with the unknowns allocated in the centres. Edge is formally second order accurate in space and has an efficient steady state solver where the explicit time integration is 
accelerated by FAS multigrid and with the possibility to combine the explicit time marching with a line-implicit approach ${ }^{16}$ where the grid is highly stretched.

We will use explicit time integration using an $m$-stage Runge-Kutta time stepping scheme to march Eq. (5) towards steady state. The Runge-Kutta time stepping is embedded in an outermost loop over iterations towards steady state. This approach is existing in the flow solver and used for solving time dependent problems in dual time with the $2^{\text {nd }}$ order backward difference approach. ${ }^{18,19}$ Introducing the current global time integration, an additional intermediate loop is introduced over the $N$ time levels. Another additional outermost loop is also introduced looping over the set of time blocks which is necessary unless one solves for all time levels at the same time. It can, schematically, be formulated as

\section{Loop over time blocks $l$}

Loop in dual time, index $j$

Loop over time levels, index $i$

Loop over stages, index $k$

$$
q_{i}^{k+1, j}=q_{i}^{1, j}-\alpha_{k} \Delta \tau \widetilde{R}\left(\vec{q}_{i}^{k, j}\right) ; \quad k=1, \cdots, m
$$

where $q_{i}^{k, j} \rightarrow q_{i}$ as the solution at time level $i$ converges in dual time.

Once all $N$ flow solutions have converged in dual time, the flow solution at the last time level $N-1$ is used as initial data for the next time block as described in Eq. (4). When using multigrid, the two innermost loops above are embedded in a recursive multigrid loop over the grid levels and with the Runge-Kutta integration as smoother.

The global time integration procedure has implications on the required storage of variables in the computer code. The flow solutions and residuals have to be stored for each $N$ time levels and on all multigrid levels. Furthermore, multigrid forcing functions have to be stored for each time level on coarser grids. With the specific storage of our computer code we estimate that the additional memory is about a factor of $1+N / 6$. For the examples considered here, a time block with $N=17$ leads to a memory increase with a factor four and with the largest time block $N=129$ a memory increase with about a factor of 22 . The additional memory requirement may require a higher degree of parallelization in space by domain partitioning and in time by splitting the time blocks.

\section{Numerical Results}

We apply the new time integrators initially on a model problem and later to the time dependent flow over a cylinder.

\section{Model problem}

The first problem we apply the time integrators to is an ordinary differential equation in time,

$$
\frac{d q}{d t}=\mu q ; \quad q(0)=q_{0}
$$

with the exact solution $q=q_{0} e^{\mu t}$ and where $\mu=-0.1, q_{0}=1$ has been used. A block size of $N=17$ is used and Eq. (9) is solved with various time steps starting from a time step $\Delta t_{0}=3.2$ with 4 blocks. In the computations, the time step is successively reduced with a factor of 2 , the number of blocks is increased with a factor of 2 and the block size is kept constant. The results with the four SBP operators are compared to results using BDF2 and ESDIRK64. Due to ESDIRK64 being a multistage scheme, the errors obtained with this scheme is displayed with a time step divided with 5 being the number of implicit stages. Calculations are carried out with a program using 32 digits representation to avoid the influence from round-off errors.

The decay of the errors for all involved time integrators are displayed in Fig. 1. To the left the errors, computed in L2-norm, involve all time levels whereas the right figure displays the L2 errors computed in the last time step of the SBP blocks. The results with BDF2 and ESDIRK64 are computed similarly. We notice that the $2^{\text {nd }}$ and $4^{\text {th }}$ order accurate BDF2 and ESDIRK64 schemes follow their design orders. As expected, the errors from the SBP schemes 
decay with one order higher $(s+1)$ than the errors on the boundaries when the error is measured in all time levels. When only the last time levels in the time blocks are accounted for, the decay rates follow the schemes order in the interior (2s) which is also expected with a penalty coefficient of $\sigma=-1 .{ }^{13}$ Then the two most accurate SBP schemes $(\operatorname{SBP}(6,3), \operatorname{SBP}(8,4))$ are substantially more accurate than the ESDIRK64 scheme.
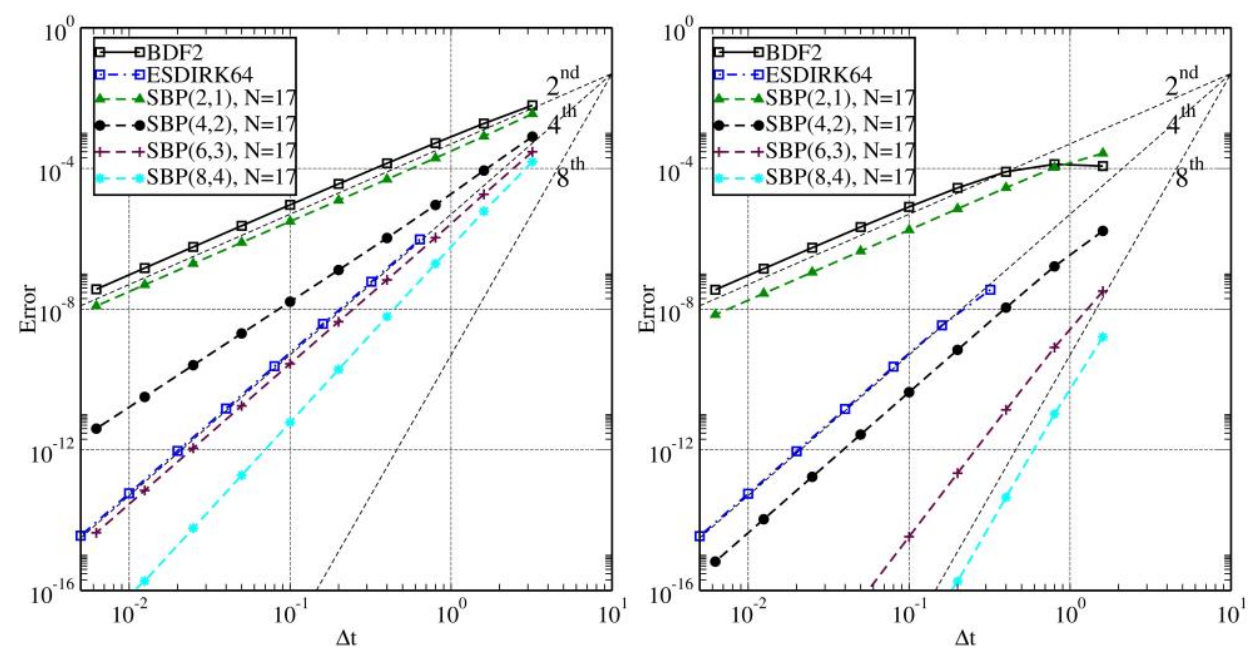

Figure 1. L2 errors for the model problem as function of number of time step size. Block sizes $N=17$. Left: Errors from all computed time levels. Right: Errors from last time level in a block.

\section{Flow over a cylinder}

Next we apply the novel time integration approach on a test case from a recent work ${ }^{20}$ where we compared the efficiency of the second order backward difference method and various diagonal implicit Runge-Kutta schemes, up to fourth order accurate in time. We compare the SBP operators with various time block sizes in terms of accuracy and efficiency.

The test case considered involves (assumed) laminar flow over a cylinder at $\mathrm{Re}=1200$ with periodic vortex shedding behind it. The Mach number is 0.3 and the same test case has been studied in the past by several authors using higher order accurate time integrators ${ }^{20-22}$ with comparisons to the $2^{\text {nd }}$ order backward difference (BDF2) scheme. The more accurate time integrators proved to be somewhat more efficient provided that the time steps were small enough.

A fine mesh of $401 \times 401$ mesh points was used for the study with a normal height of the first cell outside the wall of $5 \times 10^{-4}$ diameters. This distance gives a cell Reynolds number of $\operatorname{Re}_{C}=0.6$ with a cell aspect ratio of about 10 at the surface and a moderate normal stretching of the grid. The outer boundary is located far away from the cylinder at a distance of 250 diameters to reduce its influence on the flow and integrated forces. For the calculations we use a central discretization in space with a small amount of artificial $4^{\text {th }}$ difference numerical dissipation. Three levels of multigrid with $\mathrm{W}$-cycles are used to speed up of the rate of convergence in dual time.

The flow develops a periodic solution in time with vortex shedding. The Strouhal number approaches $S t=L /\left(U_{\infty} T\right) \approx 0.24$ with a sufficient temporal resolution, where $L$ is the cylinder diameter, $U_{\infty}$ the free stream velocity and $T$ the time period of an oscillation. All time dependent solutions are initialized from an initial solution where the periodic unsteadiness has developed and progressed periodically for about 10 periods. The Mach number contours of the initial solution are displayed in Fig. 2. The different time integrators are then applied to the initial solution and integrated in time for about 1.5 periods $T$. Various sizes of the time steps $\Delta t=T / n$ are used where $n=[10,20,40,80,160,320]$ corresponding to the number of time steps $\Delta t$ per period $T$. The solutions are compared to an "exact" solution using $n=640$, starting from the same initial solution, integrated with a $4^{\text {th }}$ order accurate diagonally implicit Runge-Kutta scheme ${ }^{20}$ and converged to machine accuracy in dual time within each stage.

We employ different block sizes in time, Table 2 summarizes the block sizes used for the calculations. The smallest block sizes are limited by the operators, we require at least one interior time level using central differences. The smallest block, $N=5$ with the $\operatorname{SBP}(2,1)$ operator, has three interior levels though. The smallest block size in common for all operators contain $N=17$ time levels, the largest blocks used contain $N=129$ levels. We compare 
the accuracy as well as the efficiency of the SBP schemes to those of the $2^{\text {nd }}$ order accurate BDF2 and $4^{\text {th }}$ order accurate ESDIRK64 schemes. To have a fair comparison, the size of the displayed time step using ESDIRK64 is divided with 5 since this scheme is a multi-stage scheme and involves 5 implicit stages. The investigations focus on comparisons with the integrated drag, similar results are obtained for the lift.

Table 2. Time block sizes $N$ employed for various SBP operators and time steps, $\Delta t=T / n$.

\begin{tabular}{lccc}
\hline \hline & $\mathbf{S B P}(\mathbf{2 , 1})$ & $\mathbf{S B P}(\mathbf{4 , 2})$ & $\mathbf{S B P}(\mathbf{6 , 3}), \mathbf{S B P}(\mathbf{8 , 4})$ \\
\hline $\mathbf{n = 1 0}$ & $5,9,17$ & 9,17 & 17 \\
$\mathbf{n}=\mathbf{2 0}$ & $5,9,17,33$ & $9,17,33$ & 17,33 \\
$\mathbf{n = 4 0}$ & $5,9,17,33,65$ & $9,17,33,65$ & $17,33,65$ \\
$\mathbf{n = 8 0}$ & $5,9,17,33,65,129$ & $9,17,33,65,129$ & $17,33,65,129$ \\
$\mathbf{n = 1 6 0}$ & $5,9,17,33,65,129$ & $9,17,33,65,129$ & $17,33,65,129$ \\
$\mathbf{n = 3 2 0}$ & $5,9,17,33,65,129$ & $9,17,33,65,129$ & $17,33,65,129$ \\
\hline \hline
\end{tabular}
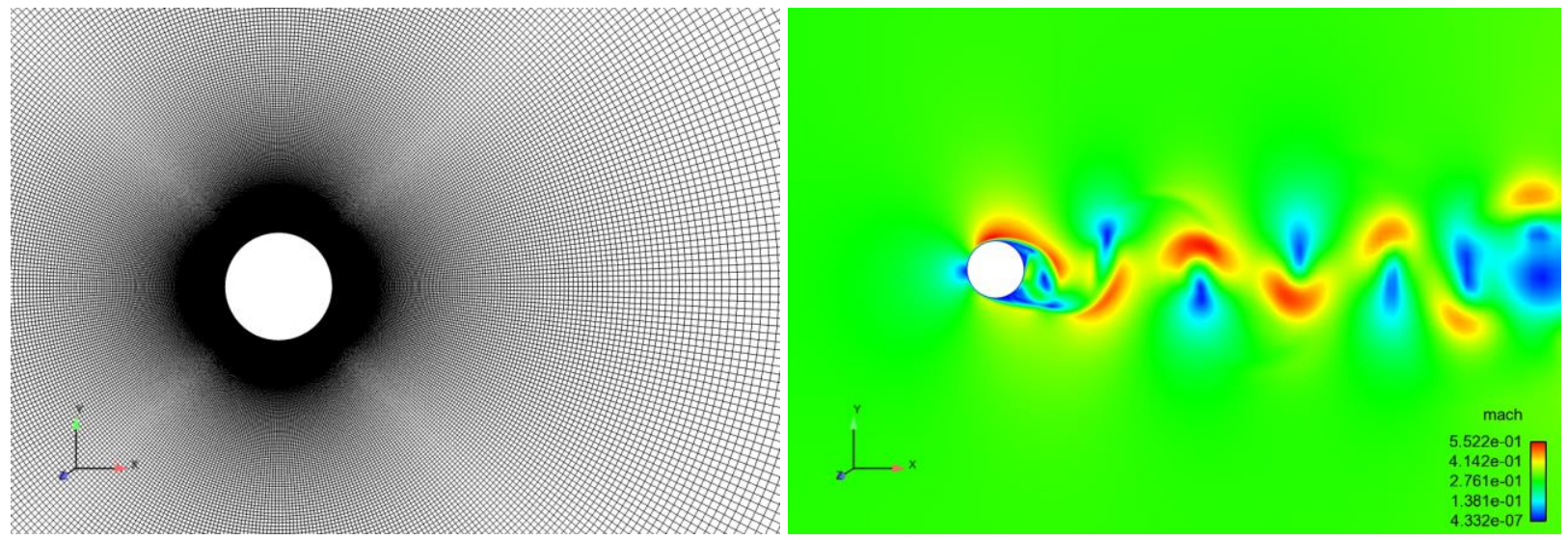

Figure 2. Laminar flow over a cylinder at $\mathbf{M = 0 . 3}, \mathbf{R e}=1200$. Left: computational near field mesh. Right: Mach number field of the initial solution.

To monitor the decay of errors we define the error as the L2norm of the difference between the current and "exact" solutions at each computed time step over the 1.5 periods of computing, i.e. the errors should correspond to the global errors.

Figure 3 displays the decay of errors in drag for all schemes as the time steps are refined and using a constant block size, $N=17$. The decay rates of the errors with BDF2 and ESDIRK64 follow the expected $2^{\text {nd }}$ and $4^{\text {th }}$ order slopes. ${ }^{20}$ The asymptotic decay rate is reached somewhere around a time step size corresponding to $n \approx 80$. The asymptotic decay rates of the errors using SBP, however, becomes higher than the expected order of accuracy. The expected orders of accuracy would be one order higher $(\mathrm{s}+1)$ than the accuracy at the boundaries since the errors are global and monitored at all time levels. The obtained overall asymptotic accuracy, however, is that of the interior $(2 \mathrm{~s})$ which is up to $8^{\text {th }}$ order with the $\operatorname{SBP}(8,4)$ scheme. This is surprising and different from the results obtained for the model problem above for which an accuracy of $2 \mathrm{~s}$ was obtained for the errors in the last time levels of the blocks only.

Figure 4 shows the decay rates for each of the SBP operators with various SBP block sizes. The overall asymptotic accuracy is the same for all block sizes and follows the accuracy of the schemes in the interior. The influence of the block size 
on the accuracy is small for all operators. There is a small but still noticeable decrease in error levels using larger blocks, the influence is largest for the more accurate SBP operators.
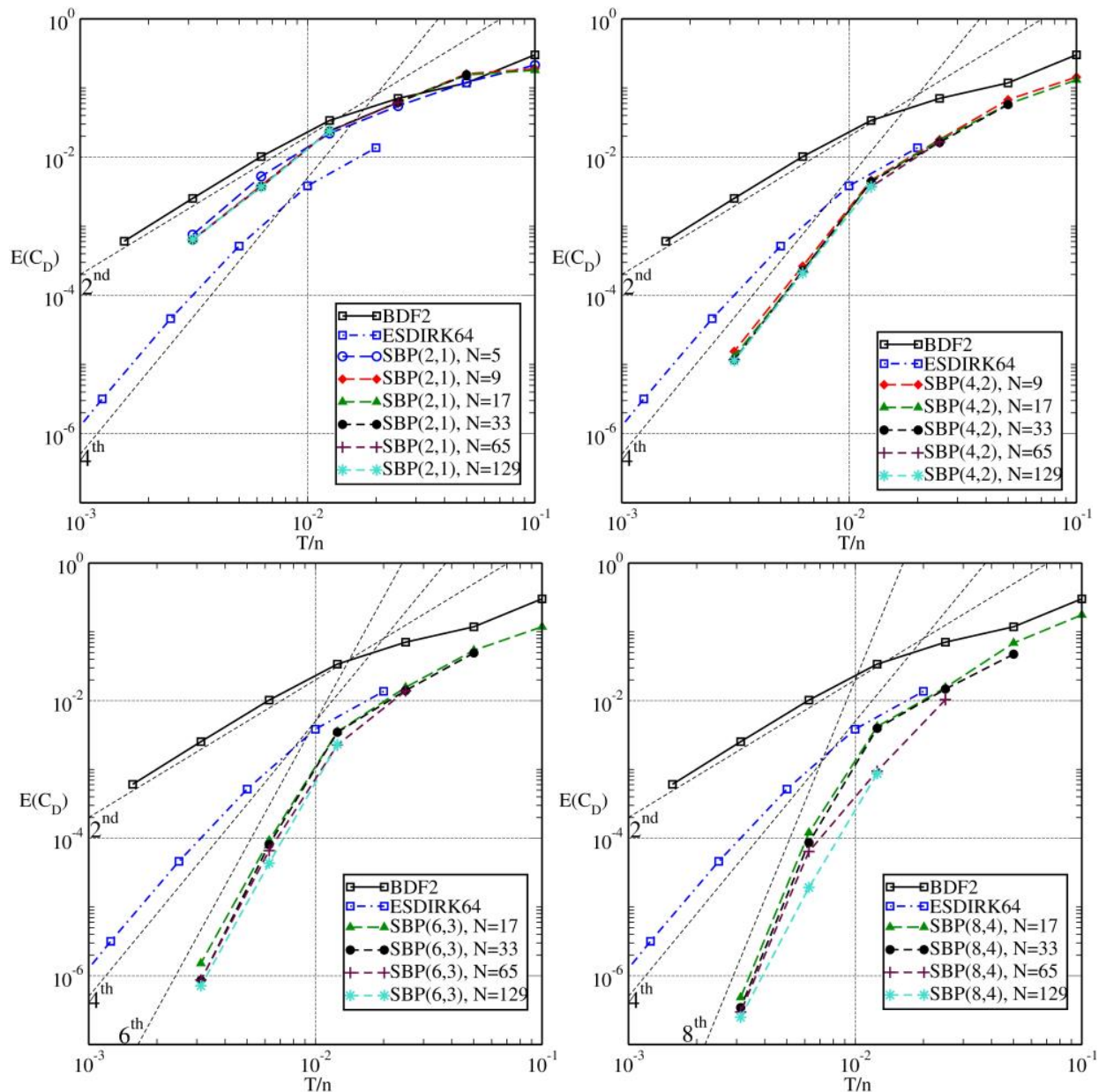

Figure 4. L2 errors of drag over 1.5 periods as function of number of time steps per period. Various block sizes $N$. Upper left: $\operatorname{SBP}(2,1)$. Upper right: $\operatorname{SBP}(4,2)$. Lower left: $S B P(6,3)$. Lower right: $\operatorname{SBP}(8,4)$.

The non-linear iterations in dual time are converged below a specified level of the maximum density residual, the same criterion is used for all time levels, and the iterations in dual time are interrupted when the residuals of all time levels satisfy the convergence criterion. Figure 5 shows the convergence of the density residual for the four SBP operators at two time levels $i \in[0, \cdots, N-1]$ considered in the first time block; the convergence of the residuals behaves similarly for all time blocks.

First of all we notice that the convergence is very similar for all SBP operator, the small differences observed may be due to different operators and coefficients for the artificial dissipation in time. An early time level in a block converges faster than a late time level that experience an initial residual increase before it starts to converge. Eventually though, both time levels decay with about the same slope. The initial increase of the residuals for the later time levels indicate that their initial solutions are further away from its converged solutions. This is due to the initialization of the solutions being the same for all time levels in a block. The solutions in the initial time block are initiated with the initial solution, all solutions in other time blocks are initiated with the solution from the last time level in the earlier time block as given in Eq. (4).

Another observation from Fig. 5 is that a smaller time step results in faster convergence at earlier time levels, compare left and right figures at the second time level $i=1$, where the residuals are converged to about 2 orders of magnitude lower levels in the right figure where the time step is only $1 / 4$ of that in the left figure. Perhaps the most important conclusion from Fig. 5 is that the required number of iterations depends mainly on the extent in time by the block and not by the size of the block. Left and right figures have different block sizes but cover about the same time and require approximately the same number of iterations. The left and mid figures, however, have the same 
block size but the mid figure advances the solution about 4 times as far in time. This also implies that smaller blocks are computationally more efficient.
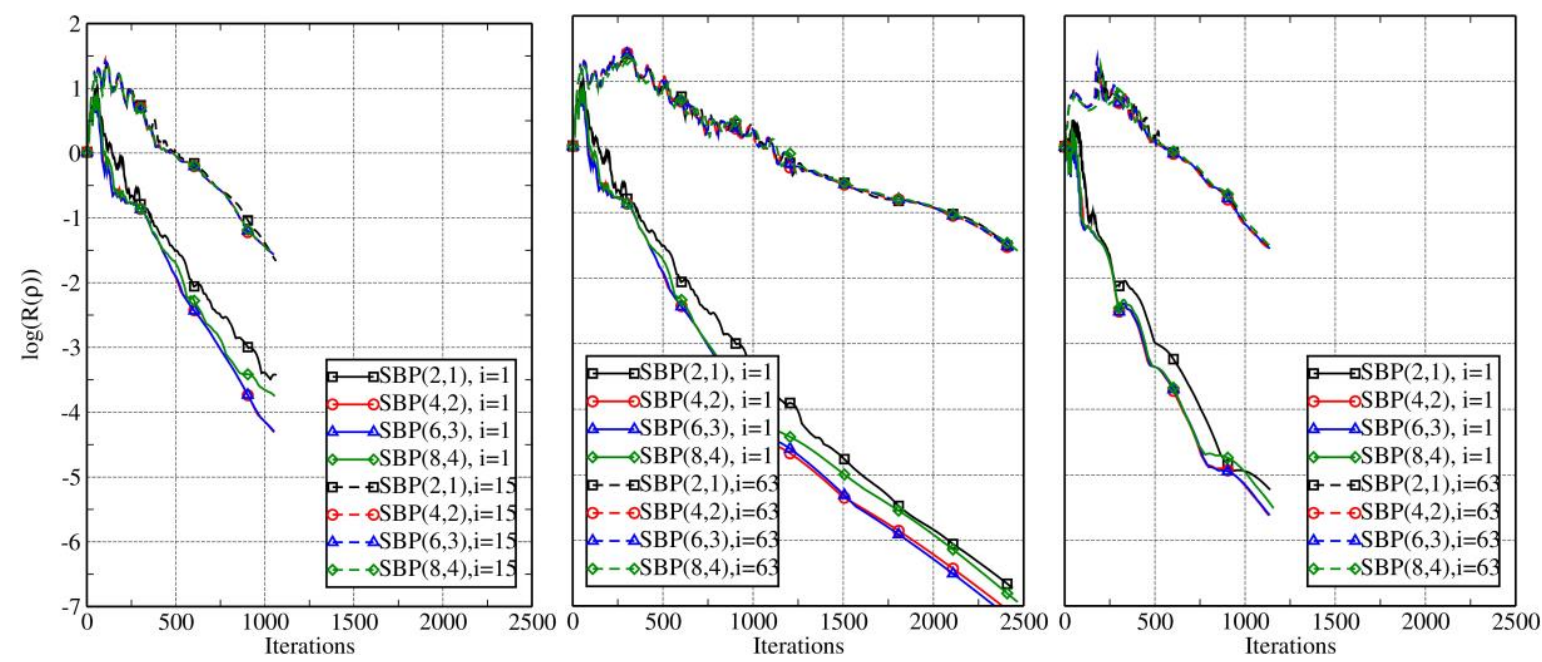

Figure 5. Convergence of density residual in dual time in the first time block for the SBP operators at two time levels using different block size and time step. Left: $\Delta t=T / 80, N=17$, time levels $i=1(t=0.0125 T)$ and $i=15$ $(t=0.187 T)$. Mid: $\Delta t=T / 80, N=65$, time levels $i=1(t=0.0125 T)$ and $i=63(t=0.787 T)$. Right: $\Delta t=T / 320, N=65$, time levels $i=1(t=0.00312 T)$ and $i=63(t=0.197 T)$.

As Fig. 5 indicates, the solution on the last time level in a block is typically the solution that converges the slowest and hence determines when to interrupt the iterations in dual time. To estimate the efficiency, we make calculations with various levels of density residual reduction to get an estimate on the required number of iterations and work. We define a solution as converged when the errors in drag have converged to within $10 \%$ of its final value. Based on this criterion, the number of iterations in dual time are estimated. We define one work unit as one multigrid cycle for a single time level.

The required average numbers of iterations per solution and time step are displayed in Fig. 6. Although the convergence curves are similar for the SBP operators, a somewhat increased level of convergence is required with more accurate operators leading to more iterations and larger required work. Smaller blocks require less iterations and are hence more efficient. A general reflection of Fig. 6 is that a fairly large number of iterations are required and that, in terms of efficiency, this can only be justified if the computed results are more accurate.

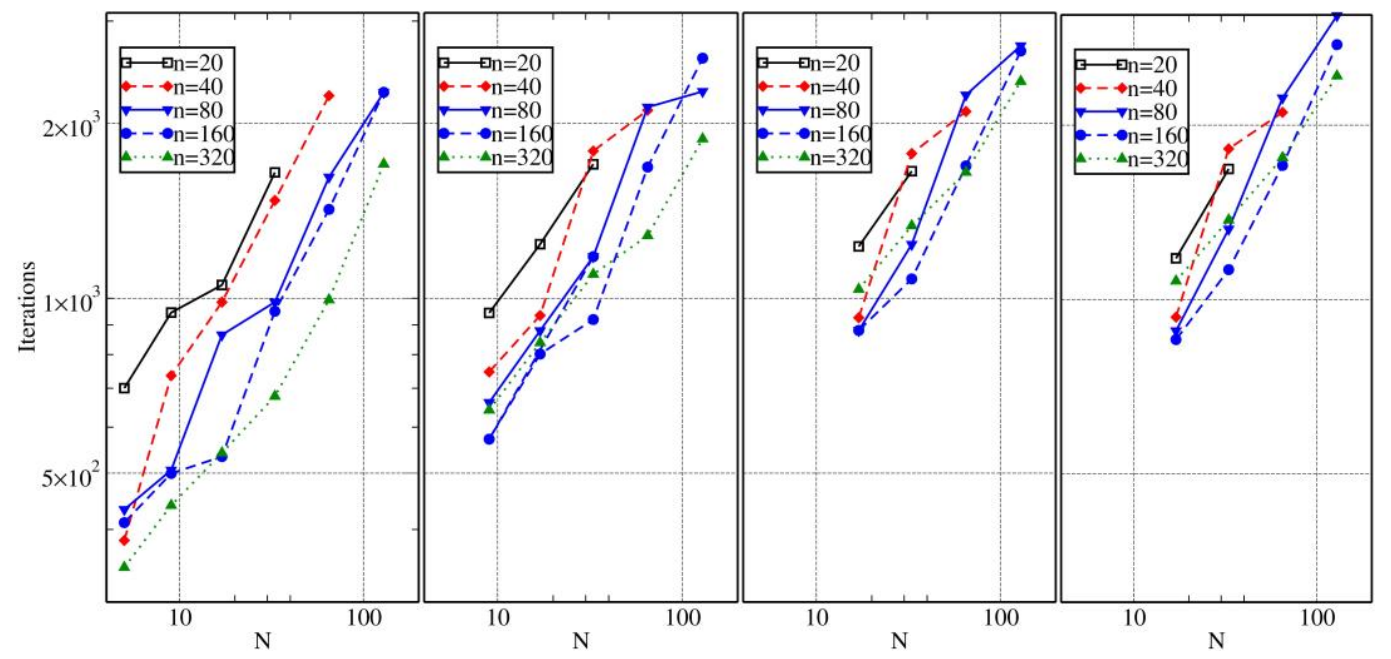

Figure 6. Required average number of iterations per solution and time step for reducing errors in drag for various block size $\mathbf{N}$. Various time steps $\Delta \mathbf{t}=\mathbf{T} / \mathbf{n}$. Left to right: $\operatorname{SBP}(2,1), \operatorname{SBP}(4,2), \operatorname{SBP}(6,3), \operatorname{SBP}(8,4)$ operators.

We estimate the efficiency by a comparison to error levels obtained with BDF2 and ESDIRK64. The BDF2 computations required about 50 iterations per time step with a very small influence from the time step size, the 
calculations with ESDIRK64 require 500 iterations or more per time step due to the 5 implicit stages. Figure 7 displays the efficiency of the SBP schemes versus those of BDF2 and ESDIRK64. The efficiency is investigated by comparing the total work (multigrid cycles) required to obtain a certain level of the error in drag, i.e. for a given error the most efficient scheme is the scheme requiring minimum work. ESDIRK64 is comparable to BDF2 for larger time steps and higher errors in drag. Reducing the time steps and errors, ESDIRK64 becomes more efficient with a higher slope.

For each time step computed and given in Table 2, the errors for all block sizes of the SBP schemes are displayed where the smallest blocks correspond to the leftmost symbols being more efficient than larger blocks. The $\operatorname{SBP}(2,1)$ scheme is not competitive compared to BDF2 since the required work is substantially higher for all time steps. As the accuracy of the SBP scheme is increased its relative efficiency increases. With the smallest time step and block size, SBP $(4,2)$ shows to have about the same efficiency as BDF2 (extrapolated to smaller time steps). ESDIRK64 is still more efficient though. The efficiency for the smallest time step and block sizes with $\operatorname{SBP}(8,4)$ is approximately the same to that of ESDIRK64.
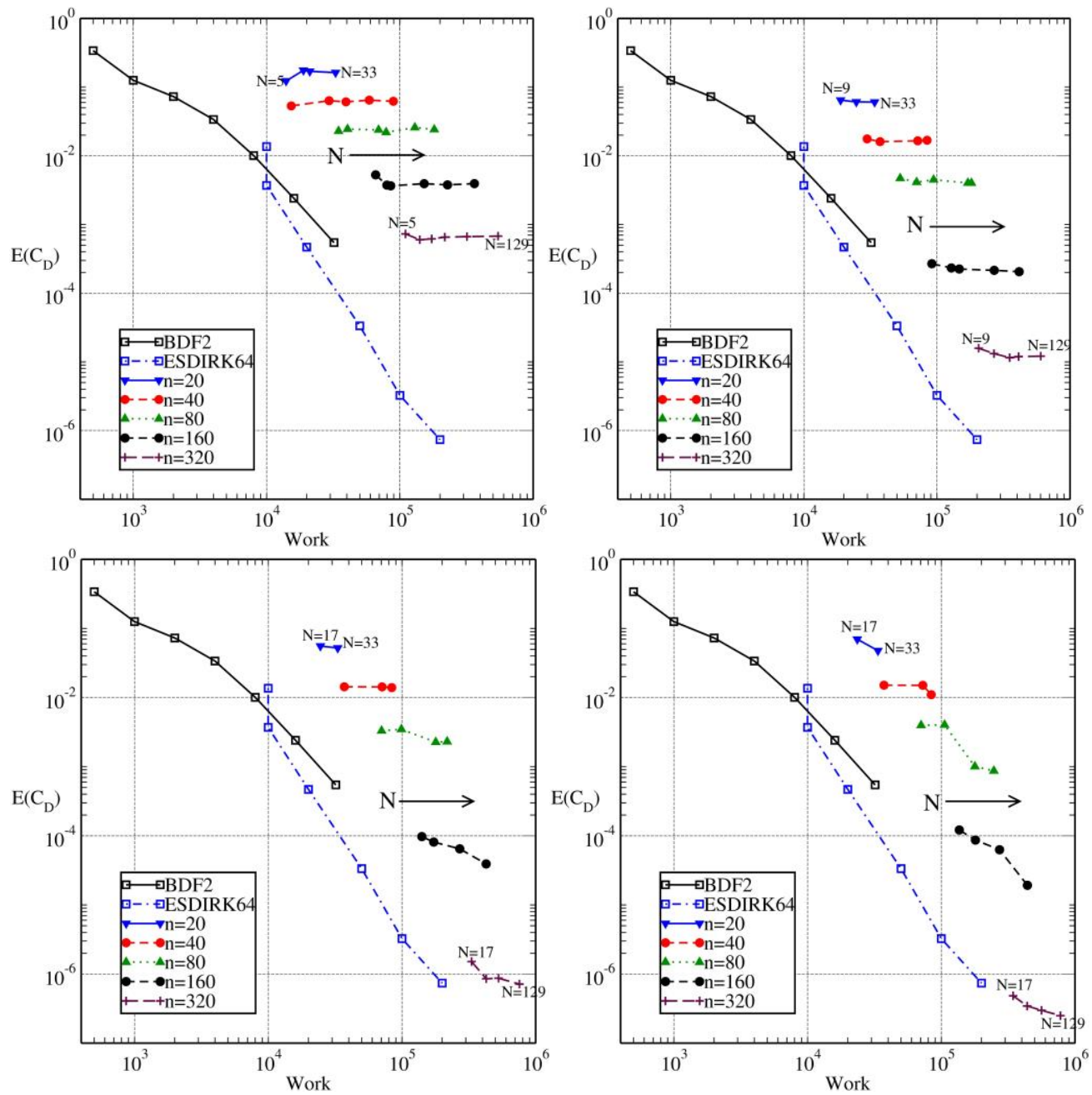

Figure 7. L2-error of integrated drag over 1.5 periods as function of work (total number of multigrid iterations). Various block size $N$ and time steps $n(\Delta t=T / n)$. Upper left: $\operatorname{SBP}(2,1)$. Upper right: $\operatorname{SBP}(4,2)$. Lower left: $\operatorname{SBP}(6,3)$. Lower right: $\operatorname{SBP}(8,4)$. 


\section{Discussion}

We present a novel time integration method for unsteady CFD calculations. The time dependent solutions are blocked in time and one solves for all flow solutions within a block simultaneously. The time discretization in a block is based on the summation-by-parts (SBP) technique in time combined with the simultaneous-approximationterm (SAT) technique for imposing the initial conditions. The time integration schemes are implicit, unconditionally stable and can be made high order accurate in time. The solutions within a time block are solved by introducing dual time stepping. To enhance convergence in dual time, artificial dissipation in time is introduced. The SBP schemes are of order $2 \mathrm{~s}$ in the interior and $\mathrm{s}$ on the time boundaries, the schemes evaluated and tested here use $\mathrm{s}=1, \ldots, 4$, the schemes are denoted $\operatorname{SBP}(2,1), \operatorname{SBP}(4,2), \operatorname{SBP}(6,3), \operatorname{SBP}(8,4)$. The expected global accuracy is s+1 making the schemes $2^{\text {nd }}, 3^{\text {rd }}, 4^{\text {th }}$ and $5^{\text {th }}$ order accurate.

The approach is first demonstrated on a model problem and compared to a conventional $2^{\text {nd }}$ order backward difference (BDF2) method and to a $4^{\text {th }}$ order accurate diagonally implicit Runge-Kutta scheme (ESDIRK64). The global accuracy $(\mathrm{s}+1)$ of the schemes is verified and it is also demonstrated that the solutions in the last time levels of the blocks are as accurate (2s) as the schemes in the interior.

The approach has been implemented in a flow solver for unstructured grids and applied to a two-dimensional unsteady flow problem. Various time block sizes and time steps have been evaluated and compared to the BDF2 and ESDIRK64 schemes in terms of accuracy and efficiency. The asymptotic accuracy of the schemes are verified and higher than expected, the accuracy follows the accuracy in the interior, up to $8^{\text {th }}$ order accuracy for the schemes considered here. The size of the blocks have a rather small influence on the overall accuracy. The different SBP schemes are most efficient for smaller blocks and the schemes become more efficient with increased accuracy of the SBP operator and small time steps. The ESDIRK64 is more efficient than BDF2 for smaller time steps and error levels. Given the current solution approach, the $\operatorname{SBP}(8,4)$ scheme with the smallest time step and block size is approximately as efficient as the ESDIRK64 scheme.

The global time integration method is based on ideas from space. There are many potential ways to further improve the efficiency of this approach, some of them may also be borrowed from approaches applied spatially like convergence acceleration techniques. One example is multigrid which is an efficient accelerator in space but could also be applied in time, and in combination, offering a more unified solution approach in space and time. Another potential improvement could be the initialization of the solutions in a new time block based on extrapolation techniques as opposed to the present approach where all flow solutions within a time block are initiated with the last solution from previous time block. Since smaller time blocks were found to be more efficient, a third approach could be to further reduce the size of the blocks with maintained accuracy by introducing a variation in the size of the time step within a block which is offered by other types of SBP operators. ${ }^{9-11}$

\section{References}

${ }^{1}$ Nordström, J., Lundquist, T. "Summation by parts in time”, Journal of Computational Physics, Vol. 251, 2013, pp. 487-499.

${ }^{2}$ Lundquist, T., Nordström, J. "The SBP-SAT technique for initial value problems", Journal of Computational Physics, Vol. 270, 2014, pp. 86-104.

${ }^{3}$ Berg, J., Nordström, J. "Stable Robin Solid Wall Boundary Conditions for the Navier-Stokes Equations", Journal of Computational Physics, Vol. 230, 2011, pp. 7519-7532.

${ }^{4}$ Carpenter, M., H., Nordström, J., Gottlieb, D. "A Stable and Conservative Interface Treatment of Arbitrary Spatial Accuracy”, Journal of Computational Physics, Vol. 148 No. 2, 1999, pp. 341-365.

${ }^{5}$ Mattsson, K., Svärd, M, Carpenter, M. and Nordström, J. “Accuracy requirements for transient aerodynamics", AIAA Paper 2003-3689, 2003.

${ }^{6}$ Svärd, M., Carpenter, M., Nordström, J., “A Stable High Order Finite Difference Scheme for the Navier-Stokes Equations, far-field Boundary Conditions", Journal of Computational Physics, Vol. 225 (1), 2007, pp. 1020-1038.

${ }^{7}$ Svärd, M., Nordström, J., "A Stable High-Order Finite Difference Scheme for the Compressible Navier-Stokes Equations: No-Slip Wall Boundary Conditions”, Journal of Computational Physics, Vol. 227, 2008, pp. 4805-4824.

${ }^{8}$ Lundquist, T., Nordström, J. "Efficient Fully Discrete Summation-by-parts Schemes for Unsteady Flow Problems", BIT Numerical Mathematics, DOI 10.1007/s10543-015-0599-0, 2015, pp. 1-16.

${ }^{9}$ Del Rey Fernandez, D., C., Boom, P., D., Zingg, D., W. "A generalized framework for nodal first derivative summation-byparts operators", Journal of Computational Physics, Vol 266, 2014, pp. 214-239.

${ }^{10}$ Boom, P., Zingg, D. "High-order implicit time-marching methods based on generalized summation-by-parts operators", Technical report, arXiv:1410.0201, 2014.

${ }^{11}$ Boom, P., Zingg, D. "High-order implicit time-marching methods based on generalized summation-by-parts operators", Technical report, arXiv:1410.0202, 2014.

${ }^{12}$ Jameson, A. “Application of Dual Time Stepping to Fully Implicit Runge Kutta Schemes for Unsteady Flow Calculations", AIAA paper 2015-2753. 
${ }^{13}$ Hicken, E., Zingg, D.W., "Dual consistency and functional accuracy: A finite-difference perspective" Journal of Computational Physics, Vol. 256, 2014, pp. 161-182.

${ }^{14}$ Mattsson, K., Svärd, M., Nordström, J. "Stable and Accurate Artificial Dissipation”, Journal of Scientific Computing, Vol. 21, No. 1, No. 1, 2004, pp. 57-79.

${ }^{15}$ Eliasson, P., Weinerfelt, P., "Recent Applications of the Flow Solver Edge", Proceedings to 7th Asian CFD Conference, Bangalore, India, 2007.

${ }^{16}$ Eliasson, P., Weinerfelt, P., Nordström, J. “Application of a Line-implicit Scheme on Stretched Unstructured Grids", AIAA-2009-0163.

${ }^{17}$ Eliasson, P., Eriksson, S., Nordström, J. "The Influence of Weak and Strong Wall Boundary Conditions on the Convergence to Steady-State of the Navier-Stokes Equations", AIAA paper 2009-3551.

${ }^{18}$ Jameson, A. "Time Dependent Calculations using Multigrid, with Applications to Unsteady Flow Past Airfoils and Wings", AIAA paper 91-1596, 1991.

${ }^{19}$ Eliasson, P., Nordström, J. "The Development of an Unsteady Solver for Moving Meshes”, Technical Report, FFA TN 1995-39.

${ }^{20}$ Eliasson, P., Weinerfelt, P. "High-Order Implicit Time Integration for Unsteady Turbulent Flow Simulations", Computers \& Fluids, 2015, Vol. 112, pp. 35-49.

${ }^{21}$ Bijl, H. Carpenter, M. H., Vatsa, V. N., Kennedy, C. A. "Implicit Time Integration Schemes for the Unsteady Compressible Navier-Stokes Equations: Laminar Flow”, Journal of Computational Physics, Vol 179, 2002, pp. 313-329.

${ }^{22}$ Jothiprasad, G., Mavriplis, D. J., Caughey, D. A. "Higher-order time integration schemes for the unsteady Navier-Stokes equations on unstructured meshes", Journal of Comp. Physics, Vol. 191, 2003, pp. 542-566.

\section{Appendix}

We present the specific forms of the stencils that satisfy the SBP stability requirements. We consider here SBP operators $q_{t} \approx D q=P^{-1} Q q$ being globally $2^{\text {nd }}$ to $5^{\text {th }}$ order accurate denoted $\operatorname{SBP}(2,1), \operatorname{SBP}(4,2), \operatorname{SBP}(6,3), \operatorname{SBP}(8,4)$ respectively. We give $P, Q$ for $\operatorname{SBP}(2,1), \operatorname{SBP}(4,2), \operatorname{SBP}(6,3)$ below.

$$
\begin{aligned}
& S B P(2,1): \quad P=\Delta t\left[\begin{array}{cccc}
1 / 2 & & & \\
& 1 & & \\
& & 1 & \\
& & & \ddots
\end{array}\right], Q=\frac{1}{2}\left[\begin{array}{cccc}
-1 & 1 & & \\
-1 & 0 & 1 & \\
& -1 & 0 & 1 \\
& & & \ddots
\end{array}\right]
\end{aligned}
$$

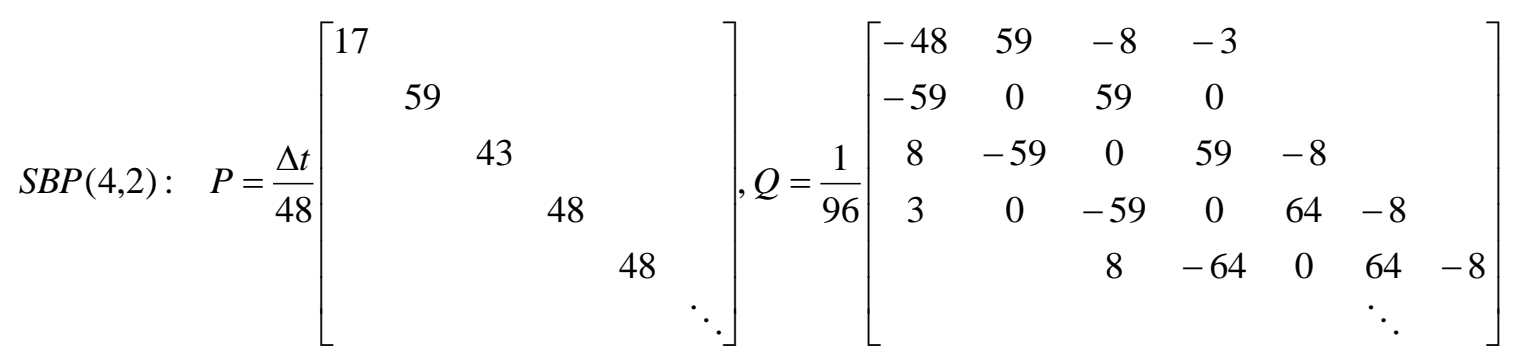

$$
\begin{aligned}
& \operatorname{SBP}(6,3): \quad P=\Delta t\left[\begin{array}{lllllll}
0.6275 & & & & & & \\
& 1.3904 & & & & & \\
& & 0.6275 & & & & \\
& & & 1.2405 & & & \\
& & & 0.9117 & & \\
& & & & 1.0139 & \\
& & & & 1 & \\
& & & & & & \ddots
\end{array}\right] \text {, }
\end{aligned}
$$




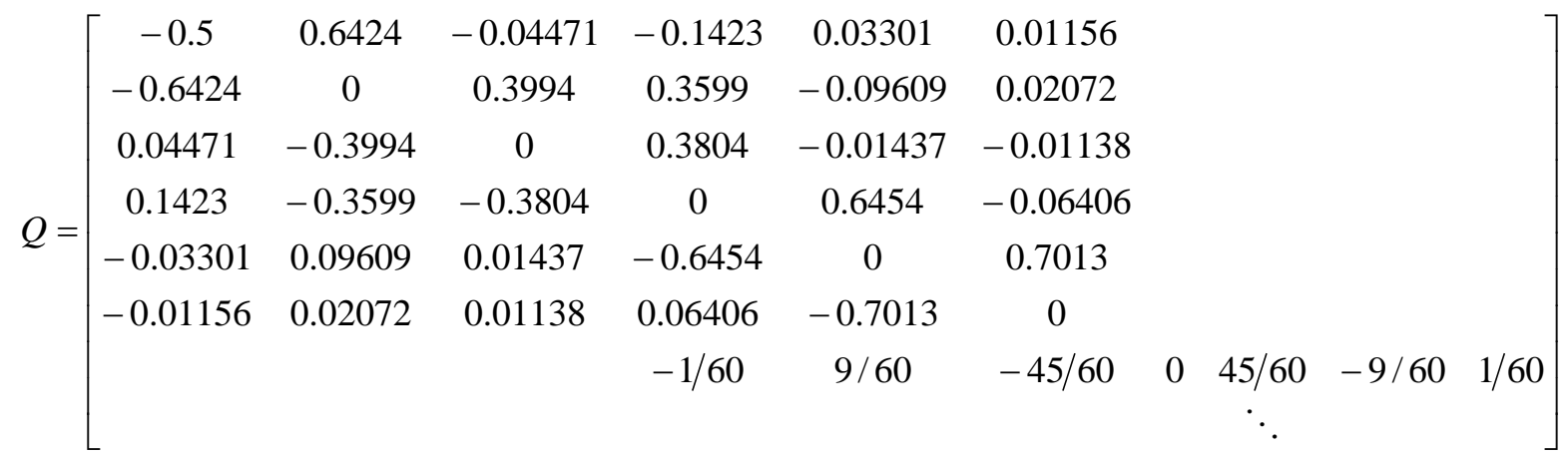

where the lower right part of the matrices are omitted. The numbers for the $\operatorname{SBP}(6,3)$ operator have been truncated to 4 digits.

Artificial dissipation in time is added as an additional term, $\varepsilon\left(P^{-1} \widetilde{D}^{T} B \tilde{D} \otimes I_{R}\right) \vec{q}$ as stated in Eq. (7). The following three combinations of operators can be employed leading to a numerical dissipation of $3^{\text {rd }}, 5^{\text {th }}$, and $7^{\text {th }}$ order accuracy, respectively:

$$
\begin{aligned}
& \tilde{D}_{2}=\left[\begin{array}{ccccc}
1 & -2 & 1 & & \\
1 & -2 & 1 & & \\
& 1 & -2 & 1 & \\
& & 1 & -2 & 1 \\
& & & & \ddots
\end{array}\right], B_{2}=\left[\begin{array}{lllll}
0 & & & & \\
& 1 & & & \\
& & 1 & & \\
& & 1 & \\
& & & \ddots
\end{array}\right], \\
& \tilde{D}_{3}=\left[\begin{array}{cccccc}
-1 & 3 & -3 & 1 & & \\
-1 & 3 & -3 & 1 & & \\
-1 & 3 & -3 & 1 & & \\
& -1 & 3 & -3 & 1 & \\
& & -1 & 3 & -3 & 1 \\
& & & & \ddots & \ddots
\end{array}\right], B_{3}=\left[\begin{array}{llllll}
0 & & & & & \\
& 0 & & & \\
& & 1 & & \\
& & 1 & & \\
& & & 1 & \\
& & & & \ddots
\end{array}\right],
\end{aligned}
$$

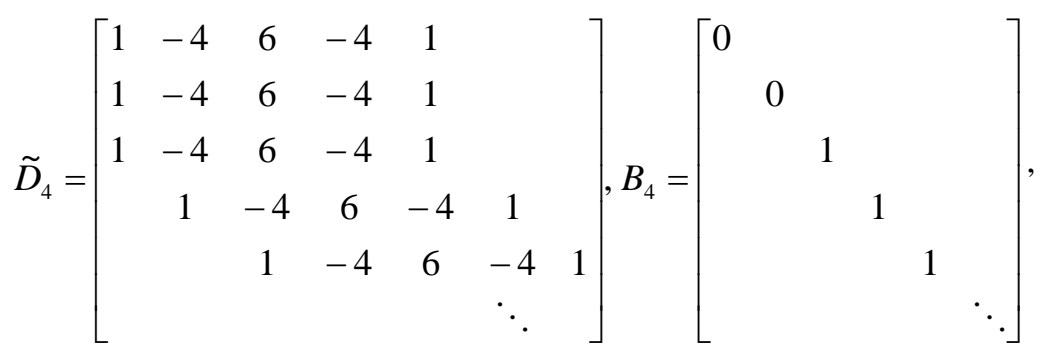

where the lower right part of the matrices are omitted. The matrix $P$ is the same operator used for the time derivative $P^{-1} Q q$. 